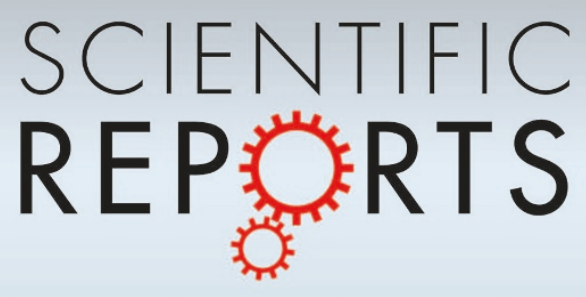

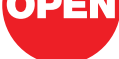

SUBJECT AREAS:

MUMERICAL DATA

MEDICAL RESEARCH

DISEASES

REPRODUCTIVE BIOLOGY

Received

22 March 2012

Accepted

20 June 2012

Published

27 July 2012

Correspondence and requests for materials should be addressed to D-K.L. (dkl@dor.kaiser.

org)

\section{A Prospective Study of In-utero Exposure to Magnetic Fields and the Risk of Childhood Obesity}

\author{
De-Kun Li ${ }^{1,2}$, Jeannette R. Ferber', Roxana Odouli ${ }^{1}$ \& Charles P. Quesenberry Jr ${ }^{1}$
}

\author{
'Division of Research, Kaiser Foundation Research Institute, Kaiser Permanente, 2000 Broadway, Oakland, California 94612, \\ ${ }^{2}$ Department of Health Research and Policy, School of Medicine, Stanford University.
}

We conducted a prospective study to examine whether in-utero exposure to magnetic fields (MFs) increases the risk of childhood obesity. Participating women carried a meter measuring MF levels during pregnancy and 733 of their children were followed up to 13 years to collect clinically recorded information on growth patterns with 33 weight measurements per child on average. Prenatal exposure to high MF level was associated with increased risk of being obese in offspring than those with lower MF level (odds ratio $=1.69$, 95\% confidence interval: 1.01-2.84). The association demonstrated a dose-response relationship and was stronger (more than 2.3 fold increased risk) among children who were followed up to the end of the study. The association existed only for persistent obesity, but not for transitory (unlikely) obesity. Maternal exposure to high MF during pregnancy may be a new and previously unknown factor contributing to the world-wide epidemic of childhood obesity/overweight.

T he prevalence of childhood obesity is increasing rapidly and has reached epidemic proportions in both industrialized and newly industrialized countries ${ }^{1-7}$. Such rapid increase signals severe public health challenges and medical care expenditures ahead, likely to increase by $\$ 48-66$ billion per year in the U.S. alone ${ }^{8}$, an alarming figure. The prevalence of obesity in US children is close to $20 \%^{1,3,9}$. The World Health Organization has considered the increased prevalence of obesity a top public health challenge ${ }^{10,11}$. While the efforts to reduce obesity have focused on changing diets and increasing physical activity with limited success, the causes of the obesity epidemic during the last several decades remain unclear.

Diet and sedentary life style cannot totally explain such a steep world-wide increase in obesity across countries with vastly differing dietary patterns and degrees of physical activity. Other environmental factors should be seriously examined, especially in pregnancy, that may damage the formation and development of fetal endocrine and metabolic systems, predisposing offspring to a higher risk of developing childhood obesity or becoming overweight $t^{10,12,13}$. Pregnancy is a critical developmental stage that is (a) among the most vulnerable periods to environmental insults, (b) susceptible to impacting multiple organ systems (an amplifying effect), and (c) more likely to result in persistent changes to an individual's adaptive physiology (long lasting consequences) ${ }^{14}$.

Among the limited studies examining environmental exposures during pregnancy on childhood obesity, impacts of physical environmental risk factors have not been examined. One such potential invisible physical risk factor is increasing man-made electromagnetic fields (EMFs) from microwave ovens to countless wireless devices. This parallel in the epidemic of obesity/overweight with massively increased use of EMF-generating appliances, and wireless networks and devices during the last a few decades warrants closer examination.

Studies have shown that EMFs could impact pregnancy outcomes and childhood diseases including asthma ${ }^{15-19}$, Higher EMF has also been associated with diabetes in humans, and overweight and high glucose level in ani$\mathrm{mals}^{20,21}$. A recent study published in JAMA showed an EMF effect on glucose metabolism, which provides a direct biological plausibility for a possible EMF effect on the risk of obesity ${ }^{22,23}$. In a recently published paper in Scientific Reports, in-utero MF exposure has been associated with ADHD in offspring indicating an EMF effect on fetal neurological development in mice ${ }^{24}$. Therefore, exposure to EMFs during pregnancy could potentially impact the fetal development including fetal endocrine and metabolic systems, predisposing offspring to a higher risk of obesity. To examine this possible association, we conducted a prospective cohort study by following a group of children (up to 13 years) whose mothers participated in a study of effect of exposure to magnetic fields (MFs) during the index pregnancy. Maternal MF exposure was measured objectively by a MF meter worn by participants during pregnancy. Children's weight information was obtained longitudinally from medical records. 


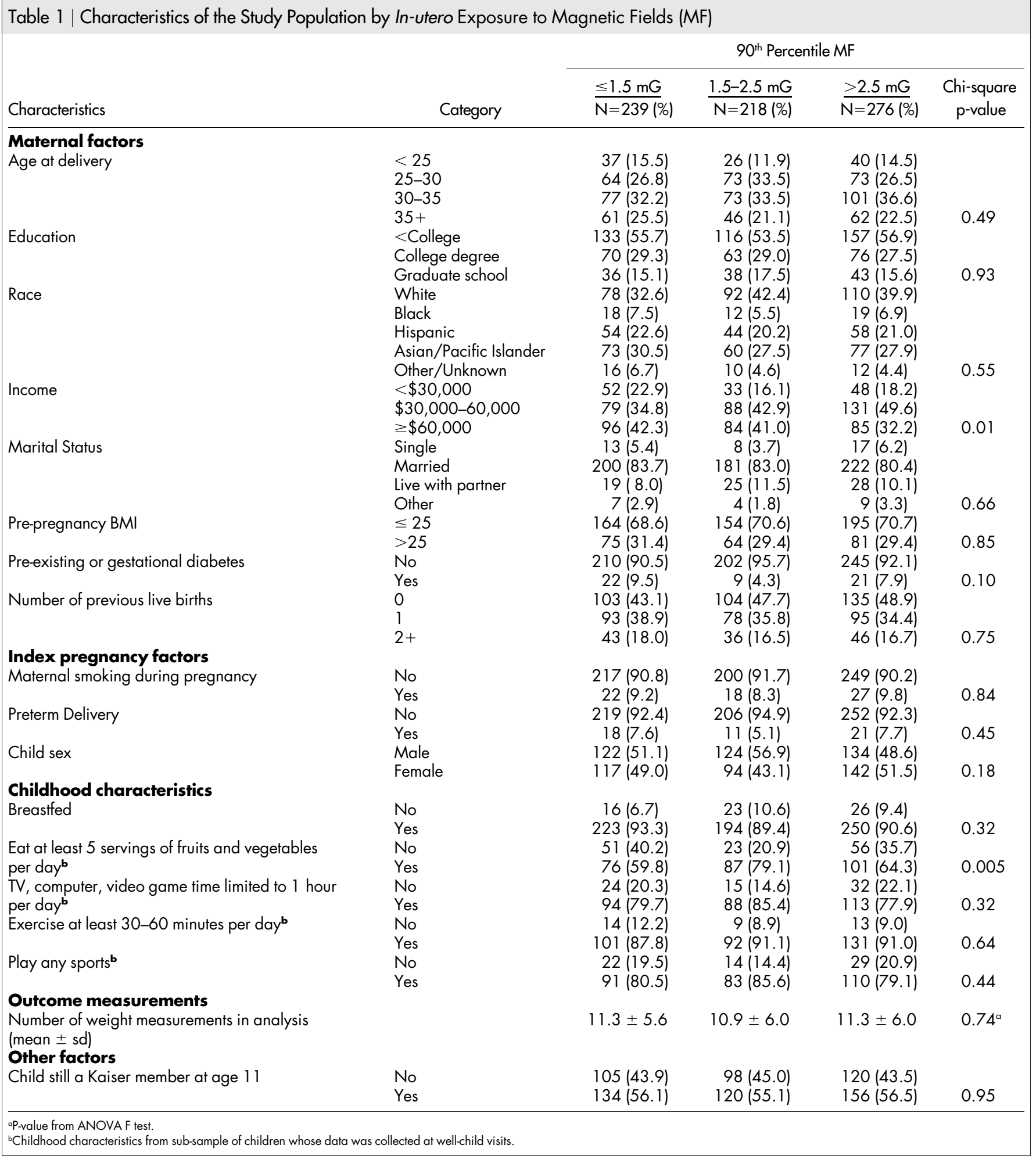

\section{Results}

Characteristics of the study population are presented in Table 1 according to in-utero MF exposure level. We examined maternal factors, prenatal factors, childhood factors, outcome measures, and other factors that may be potential confounders. Among 18 factors examined, only family income and a childhood habit of eating fruits and vegetables varied among the three maternal MF exposure groups. However, there was no consistent pattern of MF exposure with family income: women with either low or high family income had lower MF exposure level than women with medium family income. Children eating more fruits and vegetables tended to have a mother who had higher MF exposure during pregnancy. There was no difference among the three MF exposure groups in the average number of weight measurements per child. The proportion of children who remained in the KPNC system at the end of study (11 years or older) was almost identical among the three groups. 
Table 2 | In-utero Exposure to Magnetic Fields (MFs) and the Risk of Obesity/Overweight in Offspring

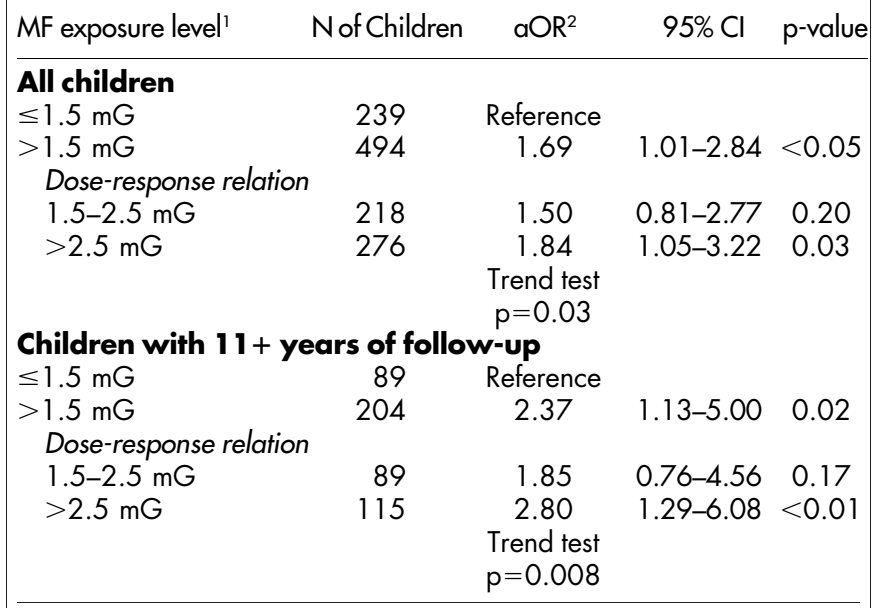

$190^{\text {th }}$ percentile of 24 hour MF exposure in milliGauss ( $\mathrm{mG}$ ).

2adjusted odd ratio of obesity/overweight defined as $>97.5$ percentile of weight-for-age based on CDC criteria ${ }^{24}$. Odds ratio from GEE model with repeated measurements, adjusted for child's exact age at each weight measurement, child gender, maternal age at delivery, maternal prepregnancy BMI, race, education level, smoking during pregnancy, and breastfeeding. Further adjustment for maternal parity, prior or gestational diabetes, income, preterm delivery, and childhood characteristics such as fruit and vegetable intake, TV watching, and exercise did not change results.

After controlling for child's age at each weight measurement, child gender, maternal age at delivery, maternal pre-pregnancy BMI, race, education level, smoking during pregnancy, and breastfeeding, inutero exposure to medium/high MF level $\left(90^{\text {th }}\right.$ percentile MF $>$ $1.5 \mathrm{mG}$ ) was associated with 69 percent increased risk of being obese or overweight during childhood $\left(>97.5^{\text {th }}\right.$ percentile the U.S age-specific weight ${ }^{25}$ ), compared to lower in-utero MF level (S $1.5 \mathrm{mG})$, adjusted odds ratio $(\mathrm{OR})=1.69,95 \%$ confidence interval (CI): 1.01-2.84 (Table 2). There was a dose-response relationship with increasing in-utero MF level being associated with increased risk of obesity/overweight: a $\mathbf{5 0 \%}$ increased risk of obesity/overweight for medium in-utero MF level (1.5-2.5 mG), and a $84 \%$ increased risk for high in-utero MF level $(>2.5 \mathrm{mG})$ with a statistically significant trend test. Additional adjustment for maternal parity, preexisting or gestational diabetes, family income, preterm delivery, and childhood behaviors such as fruit and vegetable intake, TV watching, and amount of exercise did not change the results.

Further examination of the association among the children for whom we had a complete follow-up from birth to the end of study (at least 11 years old) showed a stronger association between medium/high (vs. low) in-utero MF exposure and the risk of obesity/ overweight $(\mathrm{OR}=2.37,95 \% \mathrm{CI}: 1.13-5.00)$ as well as a stronger dose-response relationship: in-utero MF level at $1.5-2.5 \mathrm{mG}$ led to 1.85 times the risk of childhood obesity, and MF level $>2.5 \mathrm{mG}$ led to 2.80 times the risk, compared to MF level $<1.5 \mathrm{mG}$. The trend test for the dose-response relationship was highly statistically significant $(p=0.008)($ Table 2). These results indicate that a more complete follow up would have led to a stronger observed association.

To examine whether in-utero MF exposure is more likely to be associated with persistent or temporary obesity/overweight, we conducted subgroup analyses separately for children with persistent obesity/overweight and those with transitory overweight among children for whom we had complete follow-up. Children with persistent obesity throughout the follow-up period were likely to be truly obese, while those with occasional measurements reaching obesity level were less likely to have true obesity. Table 3 shows that in-utero MF exposure was much more strongly associated with persistent obesity $(\mathrm{OR}=4.97,95 \% \mathrm{CI}: 1.01-24.5)$ with a strong dose-response
Table 3 In-utero Exposure to Magnetic Fields (MFs) and the Risk of Obesity/Overweight in Offspring among Children with 11 or more years of follow-up

\begin{tabular}{|c|c|c|c|c|}
\hline MF exposure level' & $\mathrm{N}$ of Children & $\mathrm{aOR}^{4}$ & $95 \% \mathrm{Cl}$ & $\mathrm{p}$-value \\
\hline \multicolumn{5}{|c|}{ Persistent obesity/overweight ${ }^{2}$} \\
\hline$\leq 1.5 \mathrm{mG}$ & 68 & & Reference & \\
\hline$>1.5 \mathrm{mG}$ & 168 & 4.97 & 1.01 & $<0.05$ \\
\hline \multicolumn{5}{|c|}{ Dose-response relation } \\
\hline $1.5-2.5 \mathrm{mG}$ & 76 & 3.35 & $0.56-2$ & 0.19 \\
\hline$>2.5 \mathrm{mG}$ & 92 & 0.30 & $1.21-$ & 3 \\
\hline \multicolumn{5}{|c|}{ Transitory obesity/overweight $^{3}$} \\
\hline$\leq 1.5 \mathrm{mG}$ & $87 \quad R$ & eference & & \\
\hline$>1.5 \mathrm{mG}$ & 186 & 0.84 & $0.43-1.63$ & 0.61 \\
\hline \multicolumn{5}{|c|}{ Dose-response relation } \\
\hline $1.5-2.5 \mathrm{mG}$ & 82 & & 0.2 & 0 \\
\hline$>2$ & & & 0.4 & 1.00 \\
\hline \multicolumn{5}{|c|}{$\begin{array}{l}190^{\text {th }} \text { percentile of } 24 \text { hour MF exposure in milliGauss (mG). } \\
2 \geq 50 \% \text { of measurements met definition of obesity/overweight ( }>97.5 \text { percentile of weight-for-age } \\
\text { based on CDC criteria }{ }^{25} \text { ) and the child remained obese/overweight at the end of follow up (vs. } \\
\text { never overweight). } \\
{ }^{3}<50 \% \text { of measurements were above the cutoff of obesity/overweight or the child was no longer } \\
\text { obese/overweight at the end of follow up (vs. never overweight) } \\
\text { "From logistic regression model, adjusted for child gender, maternal pre-pregnancy BMl, race, } \\
\text { education level, smoking during pregnancy, and breastfeeding. Further adjustment for maternal } \\
\text { age at delivery, parity, prior or gestational diabetes, income, preterm delivery, and childhood } \\
\text { characteristics such as fruit and vegetable intake, TV watching, and exercise did not change } \\
\text { results. }\end{array}$} \\
\hline
\end{tabular}

relationship. In contrast, in-utero MF exposure was not associated with temporary obesity/overweight $(\mathrm{OR}=\mathbf{0 . 8 4}, \mathbf{9 5 \%} \mathrm{CI}$ : $0.43-1.63)$.

We also examined the interaction between in-utero MF exposure and several known risk factors for childhood obesity. Among the four risk factors we examined (high maternal pre-pregnancy BMI, maternal pre-existing or gestational diabetes, smoking during pregnancy, and a lack of breast-feeding), the MF effect on childhood obesity was noticeably stronger in the presence of all these risk factors, although the interaction with maternal smoking and a lack of breast-feeding did not reach statistical significance (Table 4). Synergetic factors are often, but not always, themselves independent risk factors ${ }^{26-28}$.

To examine the consistency of using weight and BMI measurements, we examined the association separately for weight and BMI among those who had those measurements at six years or older when BMI measurement becomes more reliable. While the number of measurements for BMI was significantly smaller than that for weight (only about 36\% weight measurements had height measurements at the same time in this subgroup), the increased risk of obesity/overweight associated with in-utero MF exposure was consistent whether weight alone or BMI was used as an outcome measure (Table 5). In addition, the association was stronger in this older age group $(\mathrm{OR}=2.49$ vs. 1.69 in all ages), probably reflecting their true obesity/overweight at older ages rather than transitory obesity/overweight at younger ages.

\section{Discussion}

In this prospective cohort study, we present a new finding that a high in-utero MF exposure level in pregnancy is associated with an increased risk of childhood obesity. The association was further supported by (a) a dose-response relationship; (b) a strong association with persistent obesity/overweight, but not with transitory overweight; and (c) interactions with known risk factors for childhood obesity: a stronger observed effect in the presence of maternal high BMI and pre-existing or gestational diabetes. Any unknown confounders would have to simultaneously account for all of these observations to explain away the association. On the other hand, these several pieces of confirmatory evidence made biological sense and pointed to an underlying association. The observed association and supporting evidence provide the first epidemiological link between 


\begin{tabular}{|c|c|c|c|c|}
\hline MF exposure level ${ }^{1}$ & $\mathrm{~N}$ of Children & $\mathrm{aOR}^{2}$ & $95 \% \mathrm{Cl}$ (p-value) & $p$-value for interaction ${ }^{3}$ \\
\hline $\begin{array}{l}\text { Maternal pre-pregnancy BMI } \\
\leq 25\end{array}$ & & & & 0.001 \\
\hline$\leq 1.5 \mathrm{mG}$ & 164 & Reference & & \\
\hline$>1.5 \mathrm{mG}$ & 349 & 1.09 & $0.54-2.21(0.81)$ & \\
\hline$>25$ & & & & \\
\hline$\leq 1.5 \mathrm{mG}$ & 75 & Reference & & \\
\hline$>1.5 \mathrm{mG}$ & 145 & 2.91 & $1.33-6.33(<0.01)$ & \\
\hline $\begin{array}{l}\text { Maternal pre-existing or gestational diabetes } \\
\text { No }\end{array}$ & & & & 0.01 \\
\hline$\leq .5 \mathrm{mG}$ & 210 & Reference & & \\
\hline$\leq 1.5 \mathrm{mG}$ & 22 & Reference & & \\
\hline$>1.5 \mathrm{mG}$ & 30 & 11.01 & $1.42-85.58(0.02)$ & \\
\hline $\begin{array}{l}\text { Smoking during pregnancy } \\
\text { No }\end{array}$ & & & & 0.19 \\
\hline$\leq 1.5 \mathrm{mG}$ & 217 & Reference & & \\
\hline$>1.5 \mathrm{mG}$ & 449 & 1.52 & $0.88-2.65(0.14)$ & \\
\hline Yes & & & & \\
\hline$\leq 1.5 \mathrm{mG}$ & 22 & Reference & & \\
\hline$>1.5 \mathrm{mG}$ & 45 & 4.87 & $1.06-22.25(0.04)$ & \\
\hline Breastfeeding & & & & 0.34 \\
\hline Yes & & & & \\
\hline$\leq 1.5 \mathrm{mG}$ & 223 & Reference & & \\
\hline$>1.5 \mathrm{mG}$ & 444 & 1.64 & $0.94-2.87(0.08)$ & \\
\hline No & & & & \\
\hline$\leq 1.5 \mathrm{mG}$ & 16 & Reference & & \\
\hline$>1.5 \mathrm{mG}$ & 49 & 2.51 & $0.36-17.40(0.35)$ & \\
\hline
\end{tabular}

$190^{\text {th }}$ percentile of 24 hour MF exposure in milliGauss $(\mathrm{mG})$.

2adjusted odd ratio of obesity/overweight defined as $>97.5$ percentile of weight-for-age based on CDC criteria ${ }^{24}$. Odds ratio from GEE model with repeated measurements, adjusted for child's exact age at each weight measurement, child gender, maternal age at delivery, maternal pre-pregnancy BMI, race, education level, smoking during pregnancy, and breastfeeding. Further adjustment for maternal parity, prior or gestational diabetes, income, preterm delivery, and childhood characteristics such as fruit and vegetable intake, TV watching, and exercise did not change results.

Interaction based on log-likelihood ratio test.

ever increasing exposure to environmental MFs (especially in-utero exposure) in the last few decades and the rapid rise in childhood obesity during the corresponding decades. Given the world-wide epidemic of childhood obesity/overweight as a leading public health challenge, our findings, if confirmed by other studies, have the potential to reveal a new environmental risk factor for childhood obesity, which may lead to prevention of childhood obesity.

While no studies have directly examined the association between MF exposure and childhood obesity, a previous study from New

Table 5 | In-utero Exposure to Magnetic Fields (MFs) and the Risk of Obesity/Overweight in Offspring over 6 Years of Age

MF exposure

$\begin{array}{llll}\text { level }^{1} \quad N \text { ofChildren } & \mathrm{aOR}^{2} & 95 \% \mathrm{Cl} & \mathrm{p} \text {-value }\end{array}$

Outcome measurements $>$ age 6

Based on weight

$\leq 1.5 \mathrm{mG}$

$>1.5 \mathrm{mG}$

Based on $B M I$

169

347

Reference
2.49

$1.22-5.07$

0.01

153

316

\section{Reference}

$\begin{array}{lll}1.87 & 0.90-3.86 & 0.09\end{array}$

$\leq 1.5 \mathrm{mG}$

$>1.5 \mathrm{mG}$

$190^{\text {th }}$ percentile of 24 hour MF exposure in milliGauss $(\mathrm{mG})$

${ }^{2}$ adjusted odd ratio of obesity/overweight defined as $>97.5$ percentile of weight/BMl-for-age based on CDC criteria ${ }^{24}$. Odds ratio from GEE model with repeated measurements, adjusted for child's exact age at each weight measurement, child gender, maternal age at delivery, maternal pre-pregnancy BMl, race, education level, smoking during pregnancy, and breastfeeding. Further adjustment for maternal parity, prior or gestational diabetes, income, preterm delivery, and childhood characteristics such as fruit and vegetable intake, TV watching, and exercise did not change results.
Zealand reported an association between high MF exposure and type II diabetes ${ }^{20}$. More recently, a study demonstrated that EMF exposure could impact cellular glucose activity level ${ }^{22}$. This finding has provided a direct possible mechanism to support our observed association. In addition, studies have shown that MF exposure during pregnancy could have impact on the fetus leading to adverse fetal outcomes and childhood diseases ${ }^{15-18}$. A recent publication in Scientific Reports showed that in-utero exposure to radio frequency EMF in mice could adversely impact neurological development in offspring $^{24}$. Therefore, it is biologically plausible that in-utero MF exposure have an adverse effect on fetal development including the metabolic and endocrine systems. Damaged metabolic and endocrine systems could lead to childhood obesity. In addition, in-utero MF exposure may be especially detrimental because the effect of environmental insults tend to be amplified both in terms of affecting multiple organ systems and having long-lasting changes to physiology such as the endocrine systems and hormone receptors ${ }^{14}$.

Compared to previous studies of EMF health effects, the current study has several strengths. The most important strength is the prospective nature of the study design. Maternal MF exposure was measured during pregnancy long before the occurrence of childhood obesity/overweight. Compared to case-control studies, the prospective study design significantly reduces participation bias due to the presence of outcomes under study. In addition, we were able to measure MF exposure prospectively during pregnancy before the onset of childhood obesity/overweight, reducing the measurement bias. The previous case-control studies of EMF health effects could only assess EMF exposure level retrospectively, usually based on recall, surrogate measures, and assumptions. EMF level is unlikely to be accurately measured retrospectively. 
Table 6 | In-utero Exposure to Magnetic Fields (MFs) and the Risk of Obesity/Overweight in Offspring, Stratified by Typical and Non-typical day

\begin{tabular}{|c|c|c|c|c|}
\hline MF exposure level ${ }^{1}$ & $\mathrm{~N}$ of Children & $\mathrm{aOR}^{2}$ & $95 \% \mathrm{Cl}$ & $p$-value \\
\hline Typical day & & & & \\
\hline$\leq 1.5 \mathrm{mG}$ & $14 /$ & Reterence & $1008 \quad 2551$ & 1006 \\
\hline $\begin{array}{l}>1.5 \mathrm{mG} \\
\text { Non-typical day }\end{array}$ & 320 & 1.80 & $\mid 0.98-3.55)$ & 0.00 \\
\hline $\begin{array}{l}\leq 1.5 \mathrm{mG} \\
>1.5 \mathrm{mG}\end{array}$ & $\begin{array}{r}92 \\
174\end{array}$ & $\begin{array}{c}\text { Reference } \\
1.59\end{array}$ & $(0.67-3.76)$ & 0.29 \\
\hline
\end{tabular}

$190^{\text {th }}$ percentile of 24 hour MF exposure in milliGauss (mG)

${ }^{2}$ adjusted odd ratio of obesity/overweight defined as $>97.5$ percentile of weight-for-age based on CDC criteria ${ }^{24}$. Odds ratio from GEE model with repeated measurements, adjusted for child's exact age at each weight measurement, child gender, maternal age at delivery, maternal prepregnancy BMI, race, education level, smoking during pregnancy, and breasffeeding. Further adjustment for maternal parity, prior or gestational diabetes, income, preterm delivery, and childhood characteristics such as fruit and vegetable intake, TV watching, and exercise did not change results.

A second strength of the study is the objective measure of both MF exposure level and weight. In most previous studies of EMF health effects, due to the retrospective nature of the study design, MF level was measured by participants' recall, surrogates (e.g., distance to power lines or current exposure to simulate past exposure), or indirect measures. These crude measures contained significant measurement errors. This type of non-differential misclassification tends to mask the underlying true association and reduce the ability to detect an existing relationship. In comparison, in this current study, pregnant mothers wore a meter that actually captured daily MF exposure from all sources, providing a much more accurate assessment of their daily MF exposure level during pregnancy. In addition, all weight measurements were obtained from medical records, rather than selfreport, providing a more accurate recording of weight information and age at the time of measurement. Also, the weight measurement was taken by practitioners without knowledge of maternal MF exposure level in pregnancy (blind).

As stated above, most children had multiple weight measurements during the follow up period (up to 13 years). The median number of weight measurements was 33 per child. Such longitudinal, multiple measurements provided rich information on weight at different ages, and also allow us to examine impact of maternal MF exposure on the different type of obesity/overweight (persistent vs. transitory). The observed strong association with persistent obesity, but no association with temporary overweight, further strengthens the argument for the underlying association between in-utero MF exposure and childhood obesity, while arguing against confounders being likely the explanation.

In addition to observing a dose-response relationship and a stronger association with persistent obesity, not with transitory overweight, we also observed a statistically significant interaction between the MF effect and two other risk factors for childhood obesity: maternal high BMI and pre-existing or gestational diabetes. Both maternal high BMI and diabetes are known risk factors for childhood obesity/overweight, and the association between in-utero MF exposure and childhood obesity was much stronger in the presence of these known risk factors. The existence of such a synergistic interaction further argues for an underlying association between in-utero MF exposure and childhood obesity.

MF measurement errors, though significantly reduced in this prospective study, might still have existed because we could only ask women to wear the meter for 24 hours during pregnancy, thus, leading to misclassification of MF exposure. Given the prospective study design, the non-differential misclassification (i.e., the same degree of misclassification to everyone) would generally lead to attenuation of observed associations. Without such misclassification, the observed association would have been even stronger. To test this hypothesis, we conducted a sensitivity analysis by stratifying on whether the MF measurement was conducted during a typical day or a non-typical day of pregnancy. It is reasonable to expect that measurements taken on a typical day reflect the MF exposure during pregnancy more accurately, thus less non-differential misclassification, while measurements taken on a non-typical day may contain more errors. As shown in Table 6, we indeed observed that less measurement error (i.e., measured on a typical day) led to a slightly stronger observed association (odds ratio $=1.86$ ) compared to more measurement error on a non-typical day (odds ratio $=1.59$ ). Therefore, had we had less measurement errors, the observed association between in-utero MF exposure and childhood obesity/overweight would have been stronger than those presented in Table 2. The same principle applies to a lack of information on MF exposure after birth (see supplemental materials for details). Because we only had one 24 -hour measurement, we were not able to evaluate the timing effect of MF exposure during pregnancy.

Another limitation is a lack of corresponding height information for more than $60 \%$ of weight measures; thus we used weight as a primary measure for overweight/obesity. However, the results in Table 5 indicate that using BMI or weight as the outcome measure showed a similar association, although BMI results did not reach statistical significance due to significantly reduced number of measurements.

In conclusion, our study provides the first epidemiologic link between in-utero MF exposure and childhood obesity/overweight. Given the worldwide epidemic of childhood obesity and ubiquitous MF exposure, this finding, if confirmed by other studies, could have implications for possibly reducing childhood obesity and understanding the obesity epidemic.

\section{Methods}

A prospective cohort study was originally conducted to examine the effect of MF exposure during pregnancy on the risk of adverse pregnancy outcomes in Kaiser Permanente Northern California (KPNC) from 1996-1998 ${ }^{16}$. The study was approved by the KPNC Institutional Review Board and informed consent was obtained from all participants.

Subject recruitment. All women who had a positive pregnancy test were identified. We recruited 1,063 women early in gestation (5-13 weeks) and 829 of them delivered a live-born infant. We excluded 28 children who did not receive pediatric care in our KPNC system.

In-person interview. Participating women were interviewed in-person during pregnancy to collect information on risk factors for adverse pregnancy outcomes, reproductive history, potential confounders and socio-demographic characteristics.

Exposure measurement: magnetic fields. EMF consists of both electric and magnetic fields. Because we used EMDEX-II meters that measure only magnetic fields, hereafter we will refer to our exposure as magnetic fields (MF). Participating women wore an EMDEX-II meter for 24 hours to measure their actual MF exposure from all sources. The meter collected MF measurements in the frequency range of $40-$ $800 \mathrm{~Hz}$ every 10 seconds. The meter was specifically programmed only to show the time of day without displaying any actual MF exposure level so that participants were not aware of their MF exposure. This design was to prevent any changes in routine daily activities due to the MF level displayed. Participants were also asked to keep a diary of their daily activities during the measurement period. At the end of the study, each participant was asked to rate the past 24 hours as a typical or non-typical day during pregnancy. The unit of MF level was measured in milliGauss (mG). We excluded 67 without complete 24 hour MF measurements. MF measurements for more than $90 \%$ of participants were conducted during the first trimester. The median gestational age at measurement was 71 days.

Outcome measurement: longitudinal weight information in offspring. Medical charts of children were reviewed to obtain information on weight and height from birth until they either (a) left the KPNC system or (b) the end of the study period (June $31,2010)$. After excluding one child with no information on weight or height, 733 mother-infant pairs were included in the final analyses.

Most children (99.5\%) had multiple measurements for weight and height, providing more detailed information on a child's growth pattern. The median number of measurements for weight per child was 33 and the number was 13 per child for height measurements. If both weight and height were measured on the same day, body mass index (BMI) was calculated.

BMI in children is not as good a measure of obesity as in adults and height in children is not as accurately measured as weight ${ }^{9}$. In addition, there were far fewer 
BMI measurements than weight measurements; more than $60 \%$ of weight measurements would have been lost due to a lack of height information. Thus, we used weight as the primary measure for overweight/obesity. The $2000 \mathrm{CDC}$ growth charts ${ }^{29}$ were used to determine age-specific percentile in weight. To reflect the secular increase of obesity/overweight in the US population, we used $97.5^{\text {th }}$ percentile (the mid-point between $95^{\text {th }}$ and $100^{\text {th }}$ percentiles) as the cutoff for obesity leading to $12.9 \%$ being classified as obese. To verify the consistency between using weight criterion alone and using BMI, we compared classification of being obese/overweight by both criteria among a subset of children who had both measurements. For measurements over age 6 (when BMI measurement is more reliable), $98.9 \%$ of those classified as obese/overweight by the weight criterion were also classified as obese by BMI cutoff at $95^{\text {th }}$ percentile.

To avoid excessive numbers of weight measurements in a short time period, we selected one weight measurement roughly every six months (when available) to reflect a reasonable measure of growth pattern while reducing unnecessary measurement variation within a short time period due to redundancy.

To separate those children with persistent obesity/overweight throughout their life from those children with temporary or transitory overweight, we considered children who were more likely to be truly obese (i.e., persistent) if (a) at least $50 \%$ of their measurements throughout the follow-up period were above $97.5^{\text {th }}$ percentile cutoff for obesity, and (b) their last measurement must also meet the cutoff for obesity, indicating that they remained obese at the end of the follow-up period (at least 11 years old).

Potential Confounders. Although MF exposure is not related to commonly known social, demographic and behavioral factors ${ }^{16}$, we evaluated many of these variables to verify. Information on some childhood behaviors such as eating habits, TV watching time, and amount of exercise was only available for a subset of children (42-46\%) from the KPNC electronic medical records. Thus, the evaluation of potential confounders for childhood factors was conducted among the subset of participants with such information available.

Data analysis. Logistic regression for repeated measurements was used to obtain point and interval estimates of association (odds ratios) after controlling for confounders. Regression coefficients and associated robust standard error estimates were estimated via generalized estimating equations, accounting for the nonindependence of the multiple longitudinal measurements per child ${ }^{30}$. We assumed an autoregressive working correlation structure, given that nearby weight measurements are more highly correlated than measurements far apart. Alternative working correlation structures (i.e., exchangeable and unstructured) were examined in sensitivity analyses, and results were consistent.

We also examined the same association among a subset of children for whom we had complete follow-up of weight measurements from their birth to the end of the study (11 years or older) to verity whether a more complete follow-up leads to a better assessment of the association. We examined the relationship of in-utero MF exposure separately with persistent obesity/overweight (yes/no) and temporary overweight (yes/no) in this subgroup, using logistic regression without repeated measurement.

Children's age at each weight measurement was included in all models to ensure that age-specific weight was compared. Although most variables evaluated were not confounders, we included in the final model the common socio-demographic variables such as maternal age, education and race/ethnicity, and the main risk factors for childhood obesity/overweight such as maternal pre-pregnancy BMI and smoking during pregnancy, as well as child's gender and history of breastfeeding.

We used $90^{\text {th }}$ percentile MF level to measure maternal MF exposure during pregnancy. MF level at $90^{\text {th }}$ percentile represents the top ten percent of MF levels in the 24 hour measurement period, which is equivalent to 2.4 hours of the highest MF exposure every day. For $90^{\text {th }}$ percentile MF exposure level, we used tertiles to divide $90^{\text {th }}$ percentile MF exposure into three levels: low $(<1.5 \mathrm{mG})$, medium $(1.5-2.5 \mathrm{mG})$, high $(>2.5 \mathrm{mG})$. We first compared low vs. medium/high to determine whether maternal MF exposure level in pregnancy had any relationship with the risk of overweight/ obesity in offspring. Once the association was observed, we then separated medium and high MF levels to determine whether there was a dose-response relationship.

1. Wang, Y. \& Lobstein, T. Worldwide trends in childhood overweight and obesity. Int. J. Pediatr. Obes. 1, 11-25 (2006).

2. de Onis, M., Blossner, M. \& Borghi, E. Global prevalence and trends of overweight and obesity among preschool children. Am. J. Clin. Nutr. 92, 1257-1264 (2010).

3. Kosti, R. I. \& Panagiotakos, D. B. The epidemic of obesity in children and adolescents in the world. Cent. Eur. J. Public Health 14, 151-159 (2006).

4. Chen, C. M. Overview of obesity in Mainland China. Obes. Rev. 9 Suppl 1, 14-21 (2008).

5. Cui, Z., Huxley, R., Wu, Y. \& Dibley, M. J. Temporal trends in overweight and obesity of children and adolescents from nine Provinces in China from 19912006. Int. J. Pediatr. Obes. 5, 365-374 (2010).

6. Yang, W. et al. Prevalence of diabetes among men and women in China. N. Engl. J. Med. 362, 1090-1101 (2010).

7. Shan, X. Y. et al. Prevalence and behavioral risk factors of overweight and obesity among children aged 2-18 in Beijing, China. Int. J. Pediatr. Obes. 5, 383-389 (2010)

8. Wang, Y. C., McPherson, K., Marsh, T., Gortmaker, S. L. \& Brown, M. Health and economic burden of the projected obesity trends in the USA and the UK. Lancet 378[9793], 815-825 (2011).
9. Barlow, S. E. Expert committee recommendations regarding the prevention, assessment, and treatment of child and adolescent overweight and obesity: summary report. Pediatrics 120 Suppl 4, S164-S192 (2007).

10. Newbold, R. R., Padilla-Banks, E., Snyder, R. J., Phillips, T. M. \& Jefferson, W. N Developmental exposure to endocrine disruptors and the obesity epidemic. Reprod. Toxicol. 23, 290-296 (2007).

11. Newbold, R. R., Padilla-Banks, E., Jefferson, W. N. \& Heindel, J. J. Effects of endocrine disruptors on obesity. Int. J. Androl. 31, 201-208 (2008).

12. Newbold, R. R., Padilla-Banks, E. \& Jefferson, W. N. Adverse effects of the model environmental estrogen diethylstilbestrol are transmitted to subsequent generations. Endocrinology 147, S11-S17 (2006).

13. Newbold, R. R. Impact of environmental endocrine disrupting chemicals on the development of obesity. Hormones. (Athens.) 9, 206-217 (2010).

14. Newbold, R. R., Padilla-Banks, E., Snyder, R. J. \& Jefferson, W. N. Perinatal exposure to environmental estrogens and the development of obesity. Mol. Nutr. Food Res. 51, 912-917 (2007)

15. Li, D. K., Chen, H. \& Odouli, R. Maternal exposure to magnetic fields during pregnancy in relation to the risk of asthma in offspring. Arch. Pediatr Adolesc. Med 165, 945-950 (2011).

16. Li, D. K. et al. A population-based prospective cohort study of personal exposure to magnetic fields during pregnancy and the risk of miscarriage. epidemiology 13, 9-20 (2002).

17. Lee, G. M., Neutra, R. R., Hristova, L., Yost, M. \& Hiatt, R. A. A nested case-control study of residential and personal magnetic field measures and miscarriages. epidemiology 13, 21-31 (2002).

18. Li, D. K., Checkoway, H. \& Mueller, B. A. Electric blanket use during pregnancy in relation to the risk of congenital urinary tract anomalies among women with a history of subfertility. Epidemiol 6, 485-489 (1995).

19. Li, D. K. et al. Exposure to magnetic fields and the risk of poor sperm quality. Reprod. Toxicol. 29, 86-92 (2010).

20. Beale, I. L., Pearce, N. E., Booth, R. J. \& Heriot, S. A. Association Of Health Problems with 50-hz Magnetic Fields in Human Adults Living Near Power Transmission Lines. Journal of Australian College of Nutritional \& Environmental Medicine 20[2], 9-12 (2001).

21. Gerardi, G. et al. Effects of electromagnetic fields of low frequency and low intensity on rat metabolism. Biomagn. Res. Technol. 6, 3 (2008).

22. Volkow, N. D. et al. Effects of cell phone radiofrequency signal exposure on brain glucose metabolism. JAMA 305, 808-813 (2011).

23. Lai, H. \& Hardell, L. Cell phone radiofrequency radiation exposure and brain glucose metabolism. JAMA 305, 828-829 (2011).

24. Aldad, T. S., Gan, G., Gao, X. B. \& Taylor, H. S. Fetal radiofrequency radiation exposure from 800-1900 mhz-rated cellular telephones affects neurodevelopment and behavior in mice. Sci. Rep. 2, 312 (2012).

25. Kuczmarski, R. J., O. C. G. S et al. 2000 CDC growth charts for the United States: Methods and development. Vital Health Stat 11[246], (2002).

26. Rothman, K. J., Greenland, S. \& Lash, T. L. Modern Epidemiology (Wolters Kluwer, Lippincott Williams \& Wilkins, 2008).

27. VanderWeele, T. J. \& Robins, J. M. The identification of synergism in the sufficient-component-cause framework. Epidemiology. 18, 329-339 (2007).

28. Darroch, J. Biologic synergism and parallelism. Am J Epidemiol. 145, 661-668 (1997).

29. Grummer-Strawn, L. M., Reinold, C. \& Krebs, N. F. Use of World Health Organization and CDC growth charts for children aged 0-59 months in the United States. MMWR Recomm. Rep. 59, 1-15 (2010).

30. Hosmer, D. W. \& Lemeshow, S. Logistic regression, $2^{\text {nd }}$ edition (John Wiley and Sons, Inc., New York, 2000)

\section{Acknowledgment}

This study was supported in part by a grant from the California Public Health Foundation.

\section{Author contributions}

D-K. L. (guarantor) is the Principal Investigator of the study and was responsible for the overall study design, obtaining funding, supervising data collection, directing data analyses, interpreting results, and preparing the manuscript. He had full access to all the data in the study and had final responsibility for the decision to submit for publication. J.F. performed the data analyses. R.O participated in data collection. C.Q. participated in data analyses and interpretation of the results.

\section{Additional information}

Supplementary information accompanies this paper at http://www.nature.com/ scientificreports

Competing financial interests: The authors declare no competing financial interests. License: This work is licensed under a Creative Commons Attribution-NonCommercial-ShareAlike 3.0 Unported License. To view a copy of this license, visit http://creativecommons.org/licenses/by-nc-sa/3.0/

How to cite this article: Li, D. et al. A Prospective Study of In-utero Exposure to Magneti Fields and the Risk of Childhood Obesity. Sci. Rep. 2, 540; DOI:10.1038/srep00540 (2012) 